confronter cette donnée avec le chiffre de l'extrait dégraissé, ce qui permet de contrôler, et souvent facilite les conclusions.

Toutes ces considérations ne sont applicables qu'aux laits non altérés. Lorsqu'on se trouve en présence de produits à forte acidité, voire de laits partiellement coagulés, comme le cas se produit malheureusement trop souvent au cours d'expertises contradictoires, il sera indispensable, à notre avis, de n'utiliser cette constante qu'avec la plus grande circonspection.

Si, en effet, le chiffre des chlorures peut toujours être dosé avec certitude, il n'en est pas de même de celui du lactose. car, en pareil cas, sa détermination devient particulièrement délicate à établir correctement, quelle que soit la méthode à laquelle on puisse avoir recours.

\title{
ANALYSES DE BEURRES PURS DES CHARENTES ET DU POITOU (1)
}

$\begin{array}{lcc}\text { par } & \\ \text { ANdRÉ CHOLLET } & \text { et } & \text { ANDRÉ CAMUS } \\ \text { Ingénieur agronome, } & & \text { Ingénieur agrieole, } \\ \text { Directeur de l'Ecole } & & \text { Chef de travaux de l'Institut } \\ \text { de Laiterie de Surgères. } & & \text { des Recherehes agronomiques. }\end{array}$

Une tromperie qui devient de plus en plus courante consiste à vendre sous le nom de "beurres des Charentes" ou de "beurres des Charentes et du Poitou " des beurres malaxés ayant une tout autre origine.

Pour tenter de mettre un frein à ces agissements, une partie des laiteries coopératives affiliées à l'Association centrale des Laiteries coopératives des Charentes et du Poitou a créé un syndicat : U. L. A. C. (Union des Laiteries de l'Association Centrale), qui a pour but de garantir l'origine et la qualité des beurres et aussi de réprimer les fraudes, soit qu'il s'agisse de graisses végétales ou de margarines vendues sous le nom de beurre, soit qu'il s'agisse simplement de la tromperie dont il vient d'être question.

Il nous a paru indispensable de baser les actions judiciaires qui pourront être engagées sur des analyses de beurres frais d'origine et de pureté certaines. Malheureusement, les documents sur ce sujet sont très rares ; l'analyse des beurres étant longue et assez coûteuse, ne semble pas avoir tenté beaucoup de chercheurs.

Nous avons employé la méthode officielle parce qu'elle est la plus utilisée dans les laboratoires chargés de la répression des fraudes.

Le premier tableau donne les résultats dans l'ordre de dates des analyses, les chiffres extrêmes étant en caractères gras.

(1) Paru dans le Volume jubilaire du Professeur Ch. Porcher. 
TABLEAU I.

RÉSULTATS D'ANALYSE DE BEURRES CLASSÊS PAR ORDRE DE DATE.

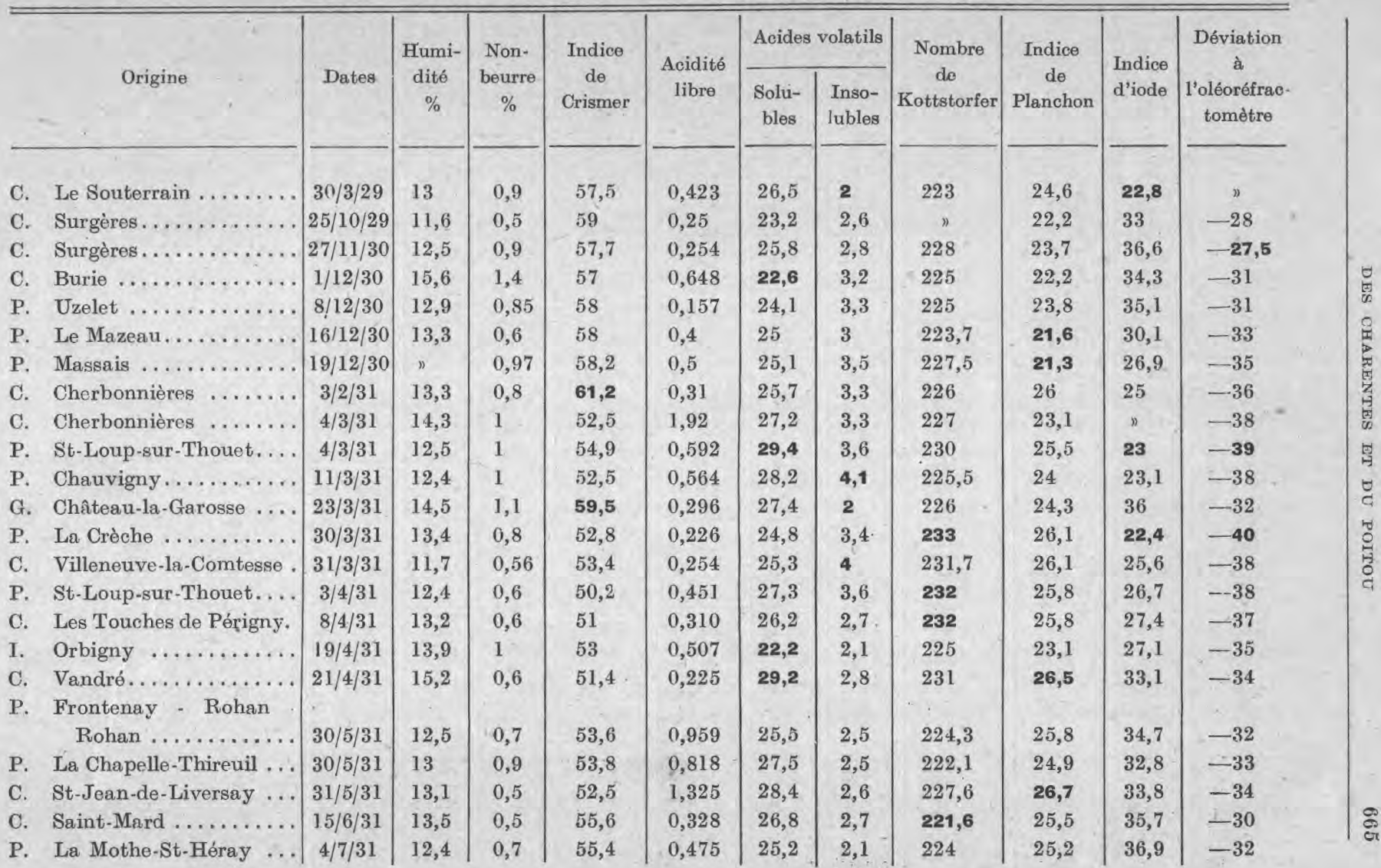

$(P=$ Poitou $=$ Deux-Sèvres, Vendée, Vienne. $)(G=$ Charente, Charente-Inférieure. $)(I=$ Indre, Indre-et-Loire. $)-(G=G i r o n d e$.$) .$ 
TABLEAU I (suite et fin).

\begin{tabular}{|c|c|c|c|c|c|c|c|c|c|c|c|}
\hline \multirow{2}{*}{ Origine } & \multirow{2}{*}{ Dates } & \multirow{2}{*}{$\begin{array}{c}\text { Humi- } \\
\text { dité } \\
\%\end{array}$} & \multirow{2}{*}{$\begin{array}{c}\text { Non. } \\
\text { beurre } \\
\%\end{array}$} & \multirow{2}{*}{$\begin{array}{l}\text { Indice } \\
\text { de } \\
\text { Crismer }\end{array}$} & \multirow{2}{*}{$\begin{array}{c}\text { Acidité } \\
\text { libre }\end{array}$} & \multicolumn{2}{|c|}{ Acides volatils } & \multirow{2}{*}{$\begin{array}{c}\text { Nombre } \\
\text { de } \\
\text { Kottstorfer }\end{array}$} & \multirow{2}{*}{$\begin{array}{c}\text { Indice } \\
\text { de } \\
\text { Planchon }\end{array}$} & \multirow{2}{*}{$\begin{array}{l}\text { Indice } \\
\text { d'iode }\end{array}$} & \multirow{2}{*}{$\begin{array}{c}\text { Déviation } \\
\text { à } \\
\text { l'oléoréfrac- } \\
\text { tomètre }\end{array}$} \\
\hline & & & & & & $\begin{array}{c}\text { Solu- } \\
\text { bles }\end{array}$ & $\begin{array}{l}\text { Inso- } \\
\text { Iubles }\end{array}$ & & & & \\
\hline Bougon $\ldots \ldots \ldots \ldots$ & $4 / 7 / 31$ & 12,3 & 0,7 & 57,3 & 0,507 & 23,4 & 2,7 & $.223,4$ & 24,6 & 37,9 & -30 \\
\hline P. La Mélusine $\ldots \ldots \ldots \ldots$ & $4 / 7 / 31$ & 12,1 & 0,9 & 55,7 & 1,03 & 25,1 & 2,1 & 224,5 & 24 & 36,2 & -32 \\
\hline P. Saint-Varent $\ldots \ldots \ldots \ldots$ & $4 / 7 / 31$ & 13 & 1 & 55 & 1,184 & 26,6 & 2,1 & 224,5 & 24,6 & 39,9 & -33 \\
\hline C. La Ronde ............ & $4 / 7 / 31$ & 13,9 & 0,8 & 54,2 & $0,4.23$ & 26,8 & 3,2 & $\Sigma 26,2$ & 26,1 & 37,4 & -32 \\
\hline P Vallans .............. & $1 / 8 / 31$ & 11 & 1,2 & 56,2 & 3,045 & 25 & 2,8 & 223,6 & 23,7 & 39,8 & -38 \\
\hline P. La Chapelle-Thireuil ... & $4 / 9 / 31$ & 12 & 0,8 & 57,1 & 0,987 & 26,5 & 2 & 222 & 23,4 & 39,8 & -35 \\
\hline C. St-Jean-de-Liversay ... & $4 / 9 / 31$ & 14,5 & 1 & 55,1 & 1,776 & 24,1 & 2 & 223,6 & 24,3 & 42,7 & -34 \\
\hline P. St-Vincent-sur-Graon ... & $28 / 9 / 31$ & $n$ & 0,7 & 54,5 & 0,733 & 27,3 & 2 & $223=$ & 24 & 34,6 & -33 \\
\hline P. La Mothe-St-Héray .... & $30 / 9 / 31$ & 12,2 & $"$ & 55,1 & 0,677 & 27,6 & 2,9 & 223,6 & 25,2 & 35,6 & -34 \\
\hline P. Frontenay - Rohan - & & & & & & & & & & & \\
\hline Rohan $\ldots \ldots \ldots \ldots$ & $3 / 10 / 31$ & 13,7 & 0,4 & 54,4 & 1,156 & 27,8 & 3,2 & 223 & 23,4 & 37,6 & -32 \\
\hline C. Saint-Mard ........... & $3 / 10 / 31$ & $\checkmark$ & 0,7 & 53,9 & 0,790 & 27,9 & $"$ & 223 & 24,3 & 38,8 & -31 \\
\hline P. La Mólusine............ & $3 / 10 / 31$ & 13 & 0,7 & 53,6 & 0,761 & 26,5 & 2,9 & 223 & 23,4 & 34,1 & -33 \\
\hline C. Baignes ............. & $3 / 10 / 31$ & 13,9 & $n$ & 55,6 & 0,705 & 27,2 & 2 & 224,8 & 26,4 & 36,1 & -33 \\
\hline P. Civray $\ldots \ldots \ldots \ldots \ldots$ & $3 / 10 / 31$ & 12,4 & $n$ & 56,7 & 0,620 & 28,6 & 2 & 226 & 26,7 & 36,3 & -33 \\
\hline C. Port d'Envaux ....... & $3 / 10 / 31$ & 14,2 & $n$ & 54,1 & 1,156 & 27,5 & 3,2 & 223,6 & 25,5 & 40,5 & -34 \\
\hline C. Matha ............... & $3 / 10 / 31$ & 13 & $n$ & 55,6 & 0,489 & 27,3 & 3,9 & 223,6 & 25,8 & 40,5 & $-32,5$ \\
\hline C. Les Rivières de Beauvais & $3 / 10 / 31$ & 12 & ” & 54,8 & 0,705 & 27,6 & 2,6 & 223,6 & 24,9 & 39,8 & -32 \\
\hline C Vervant $\ldots \ldots \ldots \ldots \ldots$ & $15 / 10 / 31$ & 13 & 0,7 & 53,3 & 1,833 & 25,6 & 2,4 & 224 & 22,5 & 39,3 & -27 \\
\hline P. Saint-Varent ......... & $13 / 10 / 31$ & $n$ & 0,7 & 53,9 & 0,338 & 27,3 & 3 & 223 & 24 & 35,8 & -32 \\
\hline C. Le Souterrain .......... & $9 / 11 / 31$ & 13,2 & $"$ & 50,1 & 0,423 & $24,1^{-}$ & 3,3 & 227 & 24,6 & 34,1 & -30 \\
\hline C. La Ronde $\ldots . . . \ldots \ldots$ & $9 / 11 / 31$ & 13,9 & $n$ & 53,8 & 0,818 & 24 & 2,1 & 226 & 23,7 & 37,8 & -29 \\
\hline C. Vervant ............. & $9 / 11 / 31$ & 14 & 1,1 & 49,9 & 0,479 & 24,2 & 2,3 & 228,4 & 24 & 30,9 & -30 \\
\hline P. Comméré-Vonillé ....... & $9 / 11 / 31$ & 13,3 & " & 49,3 & 0,451 & 27 & 3,3 & 228,5 & 24,6 & 30,6 & -32 \\
\hline P. St-Loup-sur-Thouet ... & $15 / 11 / 31$ & 12 & ") & 50,5 & 0507 & 26,9 & 3 & 230,7 & 23,8 & 31,7 & -32 \\
\hline P. Frontenay - Rohan - & & & & & & & & & & & \\
\hline Rohan ............. & $23 / 11 / 31$ & 12,9 & " & 52,4 & 0,451 & 22,9 & 2,3 & 231 & 24,4 & 33,8 & -31 \\
\hline
\end{tabular}


TABLEAU II.

ANALYSES DE BEURRES A DES DATES DIFFÉRENTES DANS DIVERSES LAITERIES.

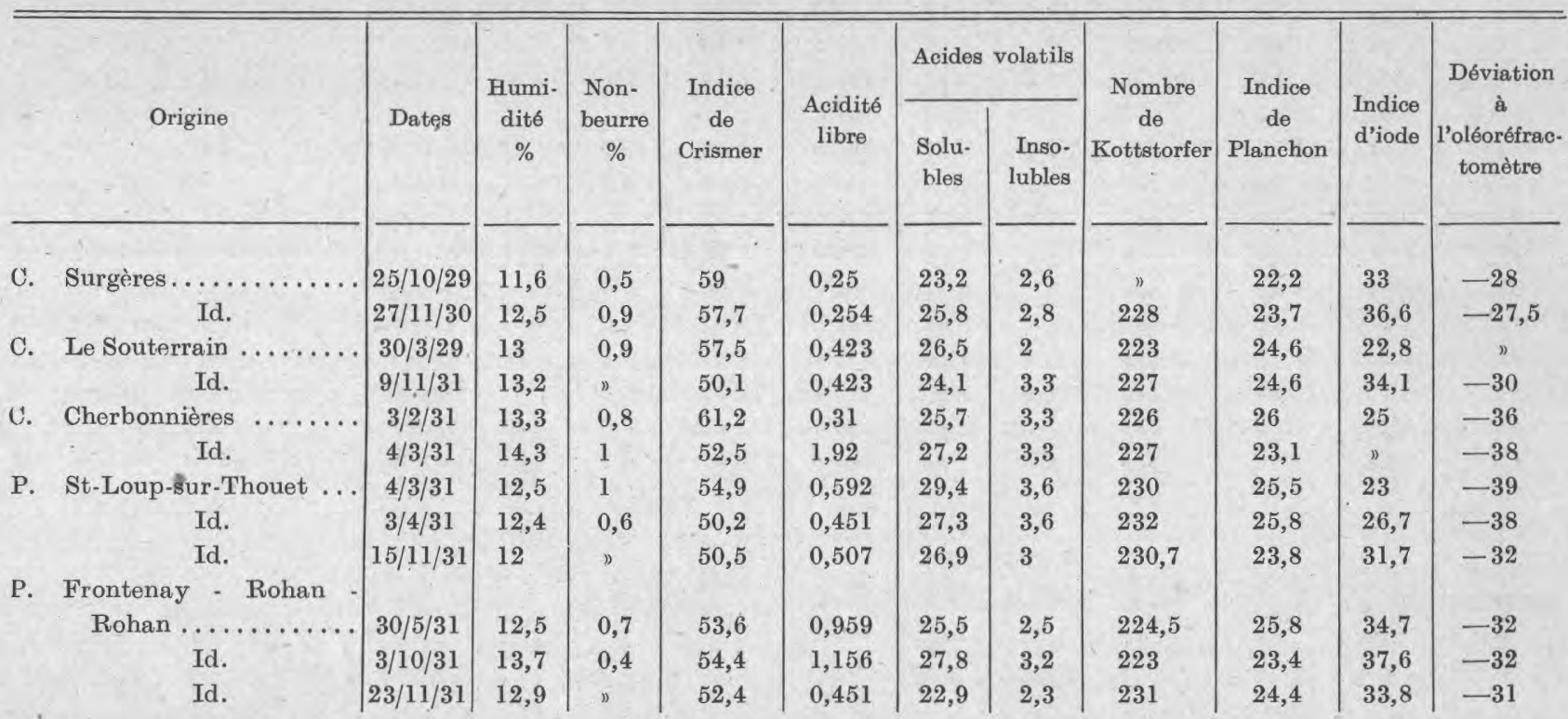


TABLEAU II (suite et fin).

\begin{tabular}{|c|c|c|c|c|c|c|c|c|c|c|c|c|}
\hline \multirow{2}{*}{\multicolumn{2}{|c|}{ Origine }} & \multirow{2}{*}{ Dates } & \multirow{2}{*}{$\begin{array}{c}\text { Humi- } \\
\text { dité } \\
\%\end{array}$} & \multirow{2}{*}{$\begin{array}{c}\text { Non- } \\
\text { beurre } \\
\%\end{array}$} & \multirow{2}{*}{$\begin{array}{c}\text { Indice } \\
\text { de } \\
\text { Crismer }\end{array}$} & \multirow{2}{*}{$\begin{array}{c}\text { Acidité } \\
\text { libre }\end{array}$} & \multicolumn{2}{|c|}{ Acides volatils } & \multirow{2}{*}{$\begin{array}{c}\text { Nombre } \\
\text { de } \\
\text { Kottstorfer }\end{array}$} & \multirow{2}{*}{$\begin{array}{c}\text { Indice } \\
\text { de } \\
\text { Planchon }\end{array}$} & \multirow{2}{*}{$\begin{array}{l}\text { Indice } \\
\text { d'iode }\end{array}$} & \multirow{2}{*}{$\begin{array}{c}\text { Déviation } \\
\text { à } \\
\text { l'oléoréfrac- } \\
\text { tomètre }\end{array}$} \\
\hline & & & & & & & $\begin{array}{l}\text { Solu- } \\
\text { bles }\end{array}$ & $\begin{array}{l}\text { Inso- } \\
\text { lubles }\end{array}$ & & & & \\
\hline \multirow[t]{2}{*}{ P. } & La Chapelle-Thireuil . & $30 / 5 / 31$ & 13 & 0,9 & 53,8 & 0,818 & 27,5 & 2,5 & 222,1 & 24,9 & 32,8 & -33 \\
\hline & Id. & $4 / 9 / 31$ & 12 & 0,8 & 57,1 & 0,987 & 26,5 & 2 & 222 & 23,4 & 39,8 & -35 \\
\hline \multirow[t]{2}{*}{ C. } & St-Jean-de-Liversay & $31 / 5 / 31$ & 13,1 & 0,5 & 52,5 & 1,325 & 28,4 & 2,6 & 227,6 & 26,7 & 33,8 & -34 \\
\hline & Id. & $4 / 9 / 31$ & 14,5 & 1 & 55,1 & 1,776 & 24,1 & 2 & 223,6 & 24,3 & 42,7 & -34 \\
\hline \multirow[t]{2}{*}{ C. } & Saint-Mard .... & $16 / 6 / 31$ & 13,5 & 0,5 & 55,6 & 0,328 & 26,8 & 2,7 & 221,6 & 25,5 & 35,7 & -30 \\
\hline & Id. & $3 / 10 / 31$ & $"$ & 0,7 & 53,9 & 0,790 & 27,9 & $"$ & 223 & 24,3 & 38,8 & -31 \\
\hline \multirow[t]{2}{*}{ P. } & La Mothe-St-Héray . & $4 / 7 / 31$ & 12,4 & 0,7 & 55,4 & 0,479 & 25,2 & 2,1 & 224 & 25,2 & 36,9 & -32 \\
\hline & Id. & $30 / 9 / 31$ & 12,2 & " & 55,1 & 0,677 & 27,6 & 2,9 & 223,6 & 25,2 & 35,6 & -34 \\
\hline \multirow[t]{2}{*}{ P. } & La Mélusine........... & $4 / 7 / 31$ & 12,1 & 0,9 & 55,7 & 1,03 & 25,1 & 2,1 & 224,5 & 24 & 36,2 & -32 \\
\hline & Id. & $3 / 10 / 31$ & 13 & 0,7 & 53,6 & 0,761 & 26,5 & 2,9 & 223 & 23,4 & 34,1 & -33 \\
\hline \multirow[t]{2}{*}{ P. } & Saint-Varent ..... & $4 / 7 / 31$ & 13 & 1 & 55 & 1,184 & 26,6 & 2,1 & 224,5 & 24,6 & 39,9 & -33 \\
\hline & Id. & $13 / 10 / 31$ & $\Rightarrow$ & 0,7 & 53,9 & 0,338 & 27,3 & 3 & 223 & 24 & 35,8 & -32 \\
\hline \multirow[t]{2}{*}{ C. } & La Ronde ...... & $4 / 7 / 31$ & 13,9 & 0,8 & 54,2 & 0,423 & 26,8 & 3,2 & 226,2 & 26,1 & 37,4 & $-3 \varepsilon$ \\
\hline & Id. & $9 / 11 / 31$ & 13,9 & $"$ & 53,8 & 0,818 & 24 & 2,1 & 226 & 23,7 & 37,8 & -29 \\
\hline \multirow[t]{2}{*}{ C. } & Vervant ....... & $15 / 10 / 31$ & 13 & 0,7 & 53,3 & 1,833 & 25,6 & 2,4 & 224 & 22,5 & 39,3 & -27 \\
\hline & Id. & $9 / 11 / 31$ & 14 & 1,1 & 49,9 & $0,4: 79$ & 24,2 & 2,3 & 228,4 & 24 & 30,9 & -30 \\
\hline
\end{tabular}


TABLEAU III

INFLUENGE DU RANCISSEMENT SUR L'ANALYSE DES BEURRES.

\begin{tabular}{|c|c|c|c|c|c|c|c|c|c|c|c|c|}
\hline \multirow[b]{2}{*}{ Origine } & \multirow[b]{2}{*}{ Dates } & \multirow{2}{*}{$\begin{array}{c}\text { Durée } \\
\text { de } \\
\text { conservation }\end{array}$} & \multirow{2}{*}{$\begin{array}{c}\text { Humi- } \\
\text { dité } \\
\%\end{array}$} & \multirow{2}{*}{$\begin{array}{l}\text { Non- } \\
\text { beurre }\end{array}$} & \multirow{2}{*}{$\begin{array}{c}\text { Indice } \\
\text { de } \\
\text { Crismer }\end{array}$} & \multirow{2}{*}{$\begin{array}{c}\text { Acidité } \\
\text { libre }\end{array}$} & \multicolumn{2}{|c|}{ Acides volatils } & \multirow{2}{*}{$\begin{array}{l}\text { Saponi- } \\
\text { fication }\end{array}$} & \multirow{2}{*}{$\begin{array}{l}\text { Indice } \\
\text { de } \\
\text { Plan- } \\
\text { chon }\end{array}$} & \multirow{2}{*}{$\begin{array}{l}\text { Indice } \\
\text { d'iode }\end{array}$} & \multirow{2}{*}{$\begin{array}{l}\text { Oléoré- } \\
\text { fracto- } \\
\text { mètre }\end{array}$} \\
\hline & & & & & & & $\begin{array}{l}\text { Solu- } \\
\text { bles }\end{array}$ & $\begin{array}{l}\text { Inso- } \\
\text { lubles }\end{array}$ & & & & \\
\hline Orbigny $\ldots \ldots \ldots \ldots$ & $19 / 4 / 31$ & Frais & 13,9 & 1 & 53 & 0,507 & 22,2 & $\varepsilon, 1$ & 225 & 23,1 & 32 & -35 \\
\hline $1 d$ & $3 / 6 / 31$ & 6 semaines & n & 1,2 & $\tilde{5} 3,2$ & 2,82 & 24,7 & ") & 224 & 22,2 & 32 & -35 \\
\hline : Id. & $28 / 9 / 31$ & 5 mois & $n$ & " & 54,8 & 6,57 & 22 & 2,3 & 222,1 & 20,7 & 31,8 & -39 \\
\hline Surgèrcs . . . . . . . . . & $=7 / 11 / 30$ & Frais & 12,5 & 0,9 & 57,7 & 0,254 & 25,8 & 2,8 & 226 & 23,7 & 36,6 & $-27,5$ \\
\hline Id. & $12 / 12 / 30$ & 15 jours & 12,5 & $n$ & 58,6 & 1,28 & 23,8 & 3,1 & 224,8 & 23,4 & 34,1 & -30 \\
\hline St-Loup-sur-Thouet . . . & $4 / 3 / 31$ & Frais & 12,5 & 1,05 & 54,9 & 0,592 & 29,4 & 3,6 & 229,8 & 25,5 & 23 & -39 \\
\hline Id. & $12 / 5 / 31$ & 2 mois & 12,3 & 1,4 & 49,5 & 2,87 & 27 & 2,9 & 229,5 & 24,9 & 23,9 & -34 \\
\hline Id. & $28 / 5 / 31$ & Lavé alcool & 12,3 & 1,4 & 50,3 & 2,00 & 25,7 & 3,4 & 228,4 & 24 & 24,9 & -34 \\
\hline Frontenay-Rohan-Rohan & $3 / 10 / 31$ & Frais & 13,7 & 0,360 & 54,4 & 1,15 & 27,8 & 3,2 & 223 & 23,4 & 37,6 & -32 \\
\hline Id. & $23 / 11 / 31$ & 7 semaines & 8 & 0,9 & 56,3 & 3,13 & 24,6 & 2,1 & 224,6 & 21,6 & 37,8 & -28 \\
\hline Vallans .............. & $28 / 9 / 31$ & 2 mois & 11 & 1,17 & 56,2 & 3,04 & 25 & 2,8 & 223,6 & 23,7 & 39,8 & -38 \\
\hline Id. & $23 / 11 / 31$ & 4 mois & 6,7 & 1,7 & 54,6 & 3,38 & 25,5 & 2,7 & 224 & 22,2 & 37,6 & -30 \\
\hline La Mélusine & $3 / 10 / 31$ & Frais & 13 & 0,7 & 53,6 & 0,76 & 26,5 & 2,9 & 223 & 23,4 & 34,1 & -33 \\
\hline Id. & $23 / 11 / 31$ & 1 mois & 9,4 & 1,3 & 53,1 & 1,83 & 24,6 & 2,6 & 225 & 22,8 & 34,3 & -30 \\
\hline St-Loup-sur-Thouet . ... & $3 / 4 / 31$ & Frais & 12,4 & 0,64 & 50,2 & 0,451 & 27,3 & 3,6 & 232 & 25,8 & 26,7 & -38 \\
\hline Id. & $16 / 11 / 31$ & 7 mois & 6 & $"$ & 48,8 & 4,68 & 26,4 & 2,5 & 231 & 23,8 & 25,4 & -35 \\
\hline La Mothe-St-Hóray . . . . . & $30 / 9 / 31$ & Frais & 12,2 & $n$ & 55,1 & 0,677 & 27,6 & 2,9 & 223,6 & 25,2 & 35,6 & -34 \\
\hline Id. & $19 / 11 / 31$ & 7 semaines & 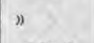 & $n$ & 53,8 & 3,04 & 25,5 & 2,3 & $"$ & 22,5 & 34,1 & -33 \\
\hline Les Touches de Périgny . & $8 / 4 / 31$ & Frais & 13,2 & 0,6 & 51 & 0,310 & 26,2 & 2,7 & 232 & 25,8 & 27,4 & -34 \\
\hline Id. & $3 / 6 / 31$ & 2 mois & $\pi$ & 0,9 & 50,7 & 2,59 & 27,4 & 3,6 & 227 & 25,2 & 29,3 & -38 \\
\hline Château-la-Garosse . & $23 / 3 / 31$ & Frais & 14,5 & 1,07 & 59,6 & 0,296 & 27,4 & 2 & 226 & 24,3 & 36 & -32 \\
\hline Id. & $8 / 5 / 31$ & 6 semaines & $D$ & $n$ & 55,7 & 4,935 & 25,1 & 1,9 & 220,6 & 23,1 & 35,4 & -32 \\
\hline Saint-Varent . . . . . . & $4 / 7 / 31$ & Frais & 13 & 1,1 & 55 & 1,184 & 26,6 & 2,1 & 225 & 24,6 & 39,9 & -33 \\
\hline Id. & $28 / 9 / 31$ & 10 semaines & 12,3 & 0,65 & 55 & 2,86 & 25,2 & 2 & 227 & 23,4 & 37,3 & -37 \\
\hline St-Jean-de-Liversay .... & $4 / 9 / 31$ & Frais . & 14,5 & 0,981 & 55,1 & 1,776 & 24,1 & 2 & 223,6 & 24,3 & 42,7 & -34 \\
\hline Id. & $23 / 11 / 31$ & 6 semaines & 7 & 1,06 & 54,7 & 3,66 & 24 & 2,4 & 224 & 21,9 & 39,6 & -27 \\
\hline
\end{tabular}


Lè second tableau, malheureusement très incomplet, rapproche les analyses d'une même laiterie faites à des époques différentes. Nous nous proposons ultérieurement de suivre pendant une année entière les variations de la composition du beurre dans une même laiterie.

D'un autre côté, les analyses des experts et surtout celle des contre-experts portent généralement, non pas sur des beurres frais, mais sur des beurres rances. La rancissure modifie-t-elle d'une façon sensible les résultats de l'analyse? Le troisième tableau rapproche les résultats obtenus dans les deux cas : analyse d'un beurre frais, analyse du même beurre rance.

Nos beurres ayant été conservés à la température du laboratoire, nous nous sommes aperçus qu'ils subissent une dessiccation notable ; cela explique en partie l'élévation du non-beurre, qui semble due également dans certains cas à la présence de mycélium de moisissures. L'indice de Crismer et l'indice de saponification varient en général assez peu. L'indice de Planchon diminue régulièrement. Les acides volatils solubles et insolubles diminuent également. Quant aux variations de l'indice d'iode et de la déviation à l'oléoréfractomètre, elles sont plus importantes, même après lavage à l'alcool dans le cas de cette dernière.

\title{
LE TRANSPORT DES LAITS ET LA FABRICATION DU GRUYERE (1).
}

\author{
CONTRIBUTION A L'ETUDE DU VIEILLISSEMENT \\ DES LAITS PAR LE TRANSPORT
}

par

L. ROY

Directeur de l'Ecole nationale d'Industrie laitière de Mamirolle (Doubs).

La fabrication du fromage de Gruyère est excessivement délicate; aussi, pour sa réussite, convient-il d'apporter un choix tout particulier aux laits à utiliser.

Dans cette matière, on peut dire que la pratique et l'expérience ont devancé la science et ont montré, bien avant que l'homme en eût - l'explication scientifique, que tous les laits étaient loin de présenter à cet égard les mêmes qualités.

L'influence de certains facteurs sur la qualité du lait a depuis longtemps déjà été mise en lumière. Les laits d'animaux fiévreux ou fatigués par exemple, riches en toxines, sont particulièrement dangereux; aussi sont-ils exclus des livraisons aux fromageries à gruyère.

(1) Paru dans le Volume jubilaire du Professeur Ch. Porcher. 